\title{
Negative to positive magnetoresistance and magnetocaloric effect in $\operatorname{Pr}_{0.6} \mathbf{E r}_{0.4} \mathbf{A l}_{2}$
}

\author{
Arjun K. Pathak, ${ }^{1, a}$ K. A. Gschneidner, Jr., ${ }^{1,2}$ and V. K. Pecharsky ${ }^{1,2}$ \\ ${ }^{1}$ The Ames Laboratory, U.S. Department of Energy, Iowa State University, Ames, Iowa \\ 50011-3020, USA \\ ${ }^{2}$ Department of Materials Science and Engineering, Iowa State University, Ames, Iowa \\ 50011-2300, USA
}

\begin{abstract}
We report on the magnetic, magnetocaloric and magnetotransport properties of $\operatorname{Pr}_{0.6} \mathrm{Er}_{0.4} \mathrm{Al}_{2}$. The title compound exhibits a large positive magnetoresistance (MR) for $\mathrm{H} \geq 40 \mathrm{kOe}$ and a small but non negligible negative $\mathrm{MR}$ for $\mathrm{H} \leq 30 \mathrm{kOe}$. The maximum positive $\mathrm{MR}$ reaches $13 \%$ at $\mathrm{H}=80 \mathrm{kOe}$. The magnetic entropy and adiabatic temperature changes as functions of temperature each show two anomalies: a broad dome-like maximum below $20 \mathrm{~K}$ and a relatively sharp peak at higher temperature. Observed behaviors are unique among other binary and mixed lanthanide compounds.
\end{abstract}

\footnotetext{
a corresponding author: pathak138@ameslab.gov
} 


\section{INTRODUCTION}

Magnetic materials that undergo temperature or field induced first order transitions attract considerable interest due to anomalous physical properties that include large magnetoresistance (MR), magnetostriction, and magnetocaloric effect (MCE) $[1,2,3,4]$. In general, electrical resistivity of magnetic materials decreases with the application of magnetic field resulting in a negative MR. In some cases, however, a positive MR has been reported in thin films [5] and bulk magnetic materials [6], but the presence of both positive and negative MR in the same material is quite rare, and is, therefore, of considerable interest from both the scientific and application points of view $[7,8]$.

Magnetism of the rare earth (R)-based $\mathrm{RAl}_{2}$ Laves phase compounds is controlled by $\mathrm{R}^{3+}$ through the indirect exchange interactions. Most of $\mathrm{RAl}_{2}$ compounds order ferromagnetically, except for $\mathrm{R}=\mathrm{Ce}, \mathrm{Eu}, \mathrm{Yb}, \mathrm{Lu}, \mathrm{La}$; their ferromagnetic $(\mathrm{FM})$ ordering temperatures, $\mathrm{T}_{\mathrm{C}}{ }^{\mathrm{s}}$, vary from $32 \mathrm{~K}$ to $180 \mathrm{~K}$ for $\mathrm{R}=\mathrm{Pr}$ and $\mathrm{Gd}$, respectively [9]. Binary rare earth dialuminides have been extensively studied, leading to a better understanding of the fundamental science and a suggestion about possible applications as low temperature magnetic refrigerants $[9,10,11,12]$. Further, interesting behaviors have been reported when two rare earth elements have been alloyed to form pseudobinary $\mathrm{R}_{1-}$ ${ }_{x} \mathrm{R}_{\mathrm{x}}^{\prime} \mathrm{Al}_{2}$ compounds. The coupling between the two rare earth ions remains FM regardless whether both $\mathrm{R}$ and $\mathrm{R}^{\prime}$ is either a heavy or a light lanthanide [13]. However, when two heavy lanthanides with oppositely shaped $4 f$ charge densities (i.e. oblate vs. prolate spheroids) are alloyed, additional anomalies have been observed in the FM state in the vicinity of the "magic" concentration $x=0.25$ in $\operatorname{Tm}_{1-\mathrm{x}} \mathrm{Tb}_{\mathrm{x}} \mathrm{Al}_{2}$ [14], $\mathrm{Er}_{1-\mathrm{x}} \mathrm{Tb}_{\mathrm{x}} \mathrm{Al}_{2}$ 
[15] and $\mathrm{Er}_{1-\mathrm{x}} \mathrm{Dy}_{\mathrm{x}} \mathrm{Al}_{2}$ [16], where the average quadrupolar moment approaches to zero [17]. On the other hand, when a light and a heavy lanthanide are combined in $\mathrm{R}_{1-\mathrm{x}} \mathrm{R}_{\mathrm{X}} \mathrm{Al}_{2}$, the moments couple antiparallel to each other [18]. The antiparallel coupling between different rare earth moments results, for example, in exchange bias in $\mathrm{Nd}_{0.75} \mathrm{Ho}_{0.25} \mathrm{Al}_{2}$ [19] and both a positive and negative $\mathrm{MCE}$ in $\mathrm{Gd}_{1-\mathrm{x}} \mathrm{Pr}_{\mathrm{x}} \mathrm{Al}_{2}$ [20].

Even though electronic transport properties of mixed light and heavy lanthanide dialuminides have not been studied, we fully expect that the presence of two magnetic ions that exhibit tendency to opposite alignment of the moments may show anomalous temperature and field dependent magnetotransport phenomena, leading to interesting physics. Here, we report anomalous behavior of magnetic, magnetotransport and magnetocaloric properties (magnetic entropy and adiabatic temperature changes) in the mixed light and heavy lanthanide rare earth dialuminide $\operatorname{Pr}_{0.6} \mathrm{Er}_{0.4} \mathrm{Al}_{2}$.

\section{EXPERIMENTAL TECHNIQUES}

A polycrystalline $\operatorname{Pr}_{0.6} \mathrm{Er}_{0.4} \mathrm{Al}_{2}$ sample was prepared by arc melting stoichiometric amounts of the constituent elements in an argon atmosphere. The Pr and Er metals were obtained from the Materials Preparation Center of the Ames Laboratory and were, respectively, $99.98+\mathrm{wt} \%(99.85+$ at. \%), and $99.98+\mathrm{wt} \%(99.82+$ at. \%) pure with respect to all other elements in the periodic table [21]. The 99.99+ wt.\% pure Al metal was purchased from Alfa Aeser Inc. The dc magnetization was measured using a vibrating sample magnetometer (Quantum Design Inc.) in magnetic fields up to $140 \mathrm{kOe}$. The heat capacity measurements were performed using a homemade adiabatic heat-pulse 
calorimeter [22] in applied magnetic fields up to $100 \mathrm{kOe}$. The electrical resistance measurements were carried out by four-terminal alternating current transport option available in the physical property measurements systems (Quantum Design Inc.) in magnetic fields up to $100 \mathrm{kOe}$. The magnetoresistance, MR, was determined from the resistivity measurements by using the relation $\mathrm{MR}=\frac{\rho(H, T)-\rho(0, T)}{\rho(0, T)} \times 100 \%$,

\section{RESULTS AND DISCUSSION}

The zero field cooled magnetization $\mathrm{M}(\mathrm{T})$ curves measured in various magnetic fields are shown in Fig. 1(a). M(T) at 20 kOe shows a weak anomaly at $\sim 24 \mathrm{~K}$, which becomes more pronounced and moves toward low temperature at $\mathrm{H} \geq 50 \mathrm{kOe}$. A clear hysteresis in the vicinity of the anomalies of magnetization during field cooled cooling and field cooled warming magnetization was observed at $\mathrm{H} \geq 50 \mathrm{kOe}$ (not shown in Fig. 1a for clarity) suggesting the first order nature of the phase transition at $\mathrm{H} \geq 50 \mathrm{kOe}$. The temperature dependence of the heat capacity $C_{p}(T)$ exhibits a single $\lambda$-like transition at $\mathrm{T}$ $=24 \mathrm{~K}$ in zero field (Fig. 1b), which is in an excellent agreement with the temperature at which a kink is observed in $\mathrm{M}(\mathrm{T})$ data measured at $\mathrm{H}=100 \mathrm{Oe}$ (Fig. 1a, inset). The monotonic decrease in magnetization and a small kink at $24 \mathrm{~K}$ (inset Fig. 1a) suggest that $\lambda$-like transition in $C_{p}(T)$ at $\mathrm{T}=24 \mathrm{~K}$ is a ferrimagnetic to paramagnetic transition. The $\lambda$-like peak shifts to lower temperature with the application of magnetic field. The upturns of $C_{p} / \mathrm{T}$ at the lowest temperatures seen in Fig. $1 \mathrm{~b}$ represent the high temperature tails of the nuclear contributions to the heat capacity of the Pr and Er atoms [23]. Quite interestingly, the observed $C_{p}$ measured in magnetic fields $50 \mathrm{kOe} \leq \mathrm{H} \leq 90 \mathrm{kOe}$ shows two 
phase transitions: a sharp first-order like low temperature peak [24], and a broad anomaly that remains centered around $\mathrm{T} \approx 22-24 \mathrm{~K}$. Earlier neutron powder diffraction data [24], as well as magnetization and heat capacity data suggested that $\operatorname{Pr}_{0.6} \mathrm{Er}_{0.4} \mathrm{Al}_{2}$ undergoes a first order ferrimagnetic (FIM) to FM transition and a second order FM to PM transition at low and high temperature, respectively. An antiparallel arrangement of light (Pr) and heavy $(\mathrm{Gd})$ lanthanide moments was also observed in $\mathrm{Gd}_{0.5} \operatorname{Pr}_{0.5} \mathrm{Al}_{2}$, by x-ray magnetic circular dichroism measurements [18]

The total entropy $S_{\text {total }}$, calculated by integrating $C_{p} / \mathrm{T}$ using equation (1), where $\mathrm{T}_{1}$ and $\mathrm{T}_{2}$ are lower and upper temperature limit of the measurements, is presented in Fig. 2.

$$
S_{\text {total }}=\int_{T_{1}}^{T_{2}} \frac{C(T)}{T} d T
$$

As shown in Fig. 2, $S_{\text {total }}$ in a zero magnetic field increases with increasing temperature and a slope change occurs at $\mathrm{T} \approx 24 \mathrm{~K}$, where the FIM state transforms to PM state. $S_{\text {total }}$ as a function of applied magnetic field shows complicated behavior: at high temperature $(\geq 30 \mathrm{~K})$, i.e. in PM state, $S_{\text {total }}$ decreases monotonically with increasing field (Fig. 2, inset b), but at low temperature $(\leq 7 \mathrm{~K}) S_{\text {total }}$ decreases with fields up to $70 \mathrm{kOe}$ and then begins to increase with a further increase of the magnetic field. The latter is related to a metamagnetic transition clearly observed at $\mathrm{H} \geq 70 \mathrm{kOe}$ (Fig. 2, insets a and b). Several jumps in the magnetization isotherm at $7 \mathrm{~K}$ (Fig. 2 inset a) along with previously reported magnetization measurements [24] suggest that this compound undergoes a series of consecutive metamagnetic transitions in magnetic fields greater than $20 \mathrm{kOe}$ below $\mathrm{T}_{\mathrm{C}}$. 
In order to evaluate the magnetocaloric properties, the isothermal magnetic entropy $\Delta S_{m}$ and the adiabatic temperature change $\Delta \mathrm{T}_{\mathrm{ad}}$ were calculated using equation (2) and (3), respectively,

$$
\Delta S_{m}(T)_{\Delta H}=S(T)_{H_{2}}-S(T)_{H_{1}}
$$

$$
\Delta T_{a d}(T)_{\Delta H}=T(S)_{H_{2}}-T(S)_{H_{1}}
$$

where $\mathrm{H}_{1}$ and $\mathrm{H}_{2}$ are lower and upper magnetic fields, respectively. The $\Delta \mathrm{S}_{\mathrm{m}}$ and $\Delta \mathrm{T}_{\mathrm{ad}}$ as functions of temperature in magnetic fields up to $100 \mathrm{kOe}$ are presented in Fig. 3a-3b. The $\Delta \mathrm{S}_{\mathrm{m}}$ and $\Delta \mathrm{T}_{\mathrm{ad}}$ exhibit two maxima: peak 1 near $25 \mathrm{~K}$ and peak 2 around $10 \mathrm{~K}$. The largest $\Delta \mathrm{S}_{\mathrm{m}}$ and $\Delta \mathrm{T}_{\text {ad }}$ in the vicinity of peak 1 for $\Delta \mathrm{H}=50 \mathrm{kOe}$ and $100 \mathrm{kOe}$ are -7.8 and $-14.7 \mathrm{~J} / \mathrm{kg} \mathrm{K}$, and 3 and $5.3 \mathrm{~K}$, respectively. As shown in Fig. 3c, the maximum $\left|\Delta \mathrm{S}_{\mathrm{m}}\right|$ and $\left|\Delta \mathrm{T}_{\mathrm{ad}}\right|$ observed at peak 1 increase steadily with increasing applied magnetic field. However, $\left|\Delta \mathrm{S}_{\mathrm{m}}\right|$ and $\left|\Delta \mathrm{T}_{\mathrm{ad}}\right|$ values at peak 2 initially increase with field up to $60 \mathrm{kOe}$ and then begin to decrease, finally merging with peak 1 when the compound exhibits only a single FM-PM transition at $\mathrm{H} \geq 90$ kOe (Fig. 1).

The temperature and field dependent resistivity, $\rho(\mathrm{H}, \mathrm{T})$, and $\mathrm{MR}$ of $\operatorname{Pr}_{0.6} \mathrm{Er}_{0.4} \mathrm{Al}_{2}$ are presented in Fig. 4. The $\rho(\mathrm{T})$ in zero magnetic field exhibits a slope change at $\mathrm{T}=24 \mathrm{~K}$ (Fig. 4a), which is in an excellent agreement with the temperature of $\lambda$-like transition observed in zero field $C_{p}(\mathrm{~T})$ data. The $\rho(\mathrm{T})$ at $40 \leq \mathrm{H} \leq 70 \mathrm{kOe}$ shows a step-like increase in resistivity with increasing temperature, where first-order transition is observed in $C_{p}(\mathrm{~T})$ (Fig. 1b), and a slope change around $24 \mathrm{~K}$. The anomaly in resistivity becomes less 
obvious when the field reaches and exceeds $80 \mathrm{kOe}$. The MR obtained from $\rho(\mathrm{T})$ data is $\approx 13 \%$ for $\Delta \mathrm{H}=80 \mathrm{kOe}$ (Fig. $4 \mathrm{c}$ ). Inset of Fig. $4 \mathrm{c}$ shows two linear dependencies of maximum MR with external magnetic fields. At low field $\mathrm{H} \leq 30 \mathrm{kOe}$, MR increases slowly with a slope of $0.8 \% / \mathrm{kOe}$ and it increases rapidly with a linear slope of 2.3 $\% / \mathrm{kOe}$ for $\mathrm{H}>30 \mathrm{kOe} . \rho(\mathrm{H})$ as a function of magnetic field initially decreases until $\mathrm{H} \approx 15$ kOe resulting in a negative MR (Fig. 4f). However, $\rho(H)$ increases rapidly when the sample undergoes first order metamagnetic transition (Fig. $4 \mathrm{~b}$ inset). The large positive MR of $13 \%$ is observed at $\mathrm{H}=80 \mathrm{kOe}$ and $\mathrm{T}=12 \mathrm{~K}$ from $\rho(\mathrm{H})$ data, which is in excellent agreement with the value obtained from $\rho(\mathrm{T})$ measurements (Fig. 4c). The low magnetic field MR in Fig. 4f shows an interesting behavior, an asymmetric MR around $\mathrm{H}$ $=0$, which is similar to that observed in spin-valve-like MR effect in other systems, e.g. $\mathrm{Mn}_{2} \mathrm{NiGa}$ [25], where the mechanism was explained by antisite disorder, in which $\mathrm{Ga}$ atom sites are partially occupied by Mn atoms forming FM nanoclusters coupled antiparallel to the other Mn atoms. The observed negative but asymmetrical MR in low magnetic field shown in Fig. 4f is most likely due to short range FM clustering and competing magnetic exchange interactions between $\mathrm{Pr}$ and Er atoms [24]. The increase in resistivity with the application of magnetic field [Fig. 4 (b-d)] is due to the antiparallel arrangement of Er and Pr moments, as suggested in Ref. [24], which could introduce spin fluctuations between FIM and FM states at high magnetic fields at low temperature. This results in a positive MR in $\operatorname{Pr}_{0.6} \mathrm{Er}_{0.4} \mathrm{Al}_{2}$ [Fig. 4c,e]. A similar effect was reported in Mnrich Ni-Mn-Ga system, where antiparallel Mn spins fluctuate with the magnetic field [26]. 


\section{CONCLUSIONS}

In conclusion, the electrical transport, thermodynamic, magnetic, and magnetocaloric properties of $\operatorname{Pr}_{0.6} \mathrm{Er}_{0.4} \mathrm{Al}_{2}$ have been studied. The $\rho(\mathrm{T}, \mathrm{H}), C_{P}(\mathrm{~T})$ and magnetization data confirm a first order metamagnetic transition, which is characterized by a jump in resistivity and $\mathrm{M}(\mathrm{H})$ measurements at $\mathrm{T}<24 \mathrm{~K}$ followed by a second order transition at high temperature. The large values of a reversible positive MR were obtained from both temperature and field dependent resistance measurements. The obtained values of $\Delta \mathrm{S}_{\mathrm{m}}$, $\Delta \mathrm{T}_{\mathrm{ad}}$, and the positive and negative MR value make $\operatorname{Pr}_{0.6} \mathrm{Er}_{0.4} \mathrm{Al}_{2}$, and other similar mixed light and heavy rare earth systems, an attractive multifunctional magnetic material.

\section{ACKNOWLEDGMENTS}

This work was supported by the U.S. Department of Energy (DOE), Office of Science, Basic Energy Sciences, Materials Science and Engineering Division. The research was performed at the Ames Laboratory, which is operated for the U.S. DOE by Iowa State University under contract \# DE-AC02-07CH11358. 


\section{Figure Captions:}

FIG. 1 (color online). (a) zero field cooled (ZFC) magnetization of $\operatorname{Pr}_{0.6} \mathrm{Er}_{0.4} \mathrm{Al}_{2}$

measured on warming in magnetic fields up to $60 \mathrm{kOe}$. The inset shows magnetization as a function of temperature at $\mathrm{H}=100 \mathrm{Oe}$. (b) The heat capacity $C_{p} / T$ as a function of temperature for $\operatorname{Pr}_{0.6} \mathrm{Er}_{0.4} \mathrm{Al}_{2}$ measured in magnetic fields up to $60 \mathrm{kOe}$.

FIG. 2 (color online). The total entropy of $\operatorname{Pr}_{0.6} \mathrm{Er}_{0.4} \mathrm{Al}_{2}$ as a function of temperature at magnetic fields up to $100 \mathrm{kOe}$. Insets (a) and (b) show zero field cooled magnetization and total entropy as a function of field at constant temperature, respectively.

FIG. 3 (color online). Magnetic entropy change, $\Delta \mathrm{S}_{\mathrm{m}}$ (a) and adiabatic temperature change, $\Delta \mathrm{T}_{\mathrm{ad}}$ (b) of $\operatorname{Pr}_{0.6} \mathrm{Er}_{0.4} \mathrm{Al}_{2}$ as a function of temperature for magnetic field changes up to $100 \mathrm{kOe}$. (c) Maximum $\left|\Delta \mathrm{S}_{\mathrm{m}}\right|$ and $\left|\Delta \mathrm{T}_{\text {ad }}\right|$ of $\operatorname{Pr}_{0.6} \mathrm{Er}_{0.4} \mathrm{Al}_{2}$ as a function of applied magnetic fields at peaks (1) $\left(\Delta \mathrm{S}_{\mathrm{m} 1} \Delta \mathrm{T}_{\mathrm{ad} 1}\right)$ and (2) $\left(\Delta \mathrm{S}_{\mathrm{m} 2} \Delta \mathrm{T}_{\mathrm{ad} 2}\right)$ shown in a and b.

FIG. 4 (Color online). The resistivity as a function of temperature, $\rho(T)$, at zero (a) and applied magnetic fields up to $100 \mathrm{kOe}(\mathrm{b})$. The inset in (b) shows the plot of $\mathrm{M}^{2}$ versus $\mathrm{H} / \mathrm{M}$ (Arrott plot) at $\mathrm{T}=12 \mathrm{~K}$. (c) The magnetoresistance, MR, as a function of temperature, obtained from data presented in (a) and (b). The inset shows peak value of MR as a function of magnetic fields, obtained from data presented in (c). (d) The resistivity as a function of applied magnetic fields, $\rho(H)$, at $T=12 \mathrm{~K}$. The $\rho(H)$ measurements were carried out as $0 \rightarrow 80 \mathrm{kOe}\left(1^{\text {st }}\right.$ cycle, close symbols $) \rightarrow 0 \mathrm{kOe}$ (reverse 
of $1^{\text {st }}$ cycle, open symbols $) \rightarrow-80 \mathrm{kOe}\left(2^{\text {nd }}\right.$ cycle, close symbols $) \rightarrow 0 \mathrm{kOe}$ (reverse of $2^{\text {nd }}$ cycle, open symbols $) \rightarrow 80$ ( $^{\text {rd }}$ cycle, close symbols $) \rightarrow 0$ kOe (reverse of $3^{\text {rd }}$ cycle, open symbols). (e) MR as a function of applied magnetic fields obtained from data presented in (d). (f) The details of MR at $-35 \mathrm{kOe} \leq \mathrm{H} \leq 35 \mathrm{kOe}$ and $\mathrm{T}=12 \mathrm{~K}$. 


\section{REFERENCES}

1 O. Tegus, E. Brück, K. H. J. Buschow and F. R. de Boer, Nature 415 (2002) 150.

${ }^{2}$ M. Apostu, R. Suryanarayanan, A. Revcolevschi, H. Ogasawara, M. Matsukawa, M. Yoshizawa, and N. Kobayashi, Phys. Rev. B 64 (2001) 012407.

${ }^{3}$ H. Tang, V. K. Pecharsky, G. D. Samolyuk, M. Zou, K. A. Gschneidner, Jr., V. P. Antropov, D. L. Schlagel, and T. A. Lograsso, Phys. Rev. Lett. 93 (2004) 237203.

${ }^{4}$ B. Maji, K. G. Suresh, and A. K. Nigam, Appl. Phys. Lett. 102 (2013) 062406.

${ }^{5}$ G. Verbanck, K. Temst, K. Mae, R. Schad, M. J. Van Bael, V. V. Moshchalkov, and Y. Bruynseraede, Appl. Phys. Lett. 70 (1997) 1477.

${ }^{6}$ S. N. Jammalamadaka, N. Mohapatra, S. D. Das, and E. V. Sampathkumaran, Phys. Rev. B 79 (2009) 060403(R).

7 B. Hu and Y. Wu, Nature Mater. 6 (2007) 985.

${ }^{8}$ H. Asano, S. B. Ogale, J. Garrison, A. Orozco, Y. H. Li, E. Li, V. Smolyaninova, C. Galley, M. Downes, M. Rajeswari, R. Ramesh, and T. Venkatesan, Appl. Phys. Lett. 74 (1999) 3696.

9 K. H. J. Buschow, Rep. Prog. Phys. 42 (1979) 1373.

10 A. K. Pathak, D. Paudyal, Y. Mudryk, K. A. Gschneidner, Jr. and V. K. Pecharsky. Phys. Rev. Lett. 110 (2013) 186405.

11 K. A. Gschneidner, H. Takeya, J. O. Moorman, and V. K. Pecharsky, Appl. Phys. Lett. 64 (1994) 253.

12 F. W. Wang, X. X. Zhang, and F. X. Hu, Appl. Phys. Lett. 77 (2000) 1360.

13 W. M. Swift and W. E. Wallace, J. Phys. Chem. Solids, 29 (1968) 2053. 
${ }^{14}$ M. Khan, D. Paudyal, Y. Mudryk, K. A. Gschneidner Jr., and V. K. Pecharsky, Phys. Rev. B 83 (2011) 134437.

15 M. Khan, Y. Mudryk, D. Paudyal, K. A. Gschneidner, Jr., and V. K. Pecharsky, Phys. Rev. B 82 (2010) 064421.

16 A. L. Lima, K. A. Gschneidner, Jr., V. K. Pecharsky, and A. O. Pecharsky, Phys. Rev. B 68 (2003) 134409.

17 D. Paudyal, A. K. Pathak, V. K. Pecharsky and K. A. Gschneidner Jr., J. Phys.: Condens. Matter 25 (2013) 396002.

18 A. Magnus G. Carvalho, F. Garcia, V. S. R. de Sousa, P. J. von Ranke, D. L. Rocco, G. D. Loula, E. J. de Carvalho, A. A. Coelho, L.M. da Silva, F. C. G. Gandra, J. Magn. Magn. Mat. 321 (2009) 3014.

19 P. D. Kulkarni, A. Thamizhavel, V. C. Rakhecha, A. K. Nigam, P. L. Paulose, S. Ramakrishnan and A. K. Grover, Eur. Phys. Lett. 86 (2009) 47003.

${ }^{20}$ V.S.R. de Sousa, A. Magnus G. Carvalho, E.J.R. Plaza, B.P. Alho, J.C.G. Tedesco, A.A. Coelho, N.A. de Oliveira, P.J. von Ranke, J. Magn. Magn. Mater. 323 (2011) 794.

21 Materials Preparation Center, Ames Laboratory of US, DOE, Ames, IA, USA.

${ }^{22}$ V. K. Pecharsky, J. O. Moorman, and K. A. Gschneidner Jr., Rev. Sci. Instrum. 68 (1997) 4196.

${ }^{23}$ L. J. Sundström, Chapter 5, Vol 1 (p. 379) in Handbook on the Physics and Chemistry of Rare Earths, edited by K. A. Gschneider, Jr. and L. Eyring (1978).

${ }^{24}$ A. K. Pathak, D. Paudyal, W. T. Jayasekara, S. Calder, A. Kreyssig, A. I. Goldman, K. A. Gschneidner, Jr., and V. K. Pecharsky, Phys. Rev. B 89 (2014) 224411. 
${ }^{25}$ S. Singh, R. Rawat, S. Esakki Muthu, S. W. D’Souza, E. Suard, A. Senyshyn, S.

Banik, P. Rajput, S. Bhardwaj, A. M. Awasthi, R. Ranjan, S. Arumugam, D. L. Schlagel, T. A. Lograsso, A. Chakrabarti, and S. R. Barman, Phys. Rev. Lett. 109 (2012) 246601.

${ }^{26}$ S. Banik, R. Rawat, P. K. Mukhopadhyay, B. L. Ahuja, A. Chakrabarti, P. L. Paulose, Sanjay Singh, A. Kumar Singh, D. Pandey, and S. R. Barman, Phys. Rev. B. 77 (2008) 224417. 

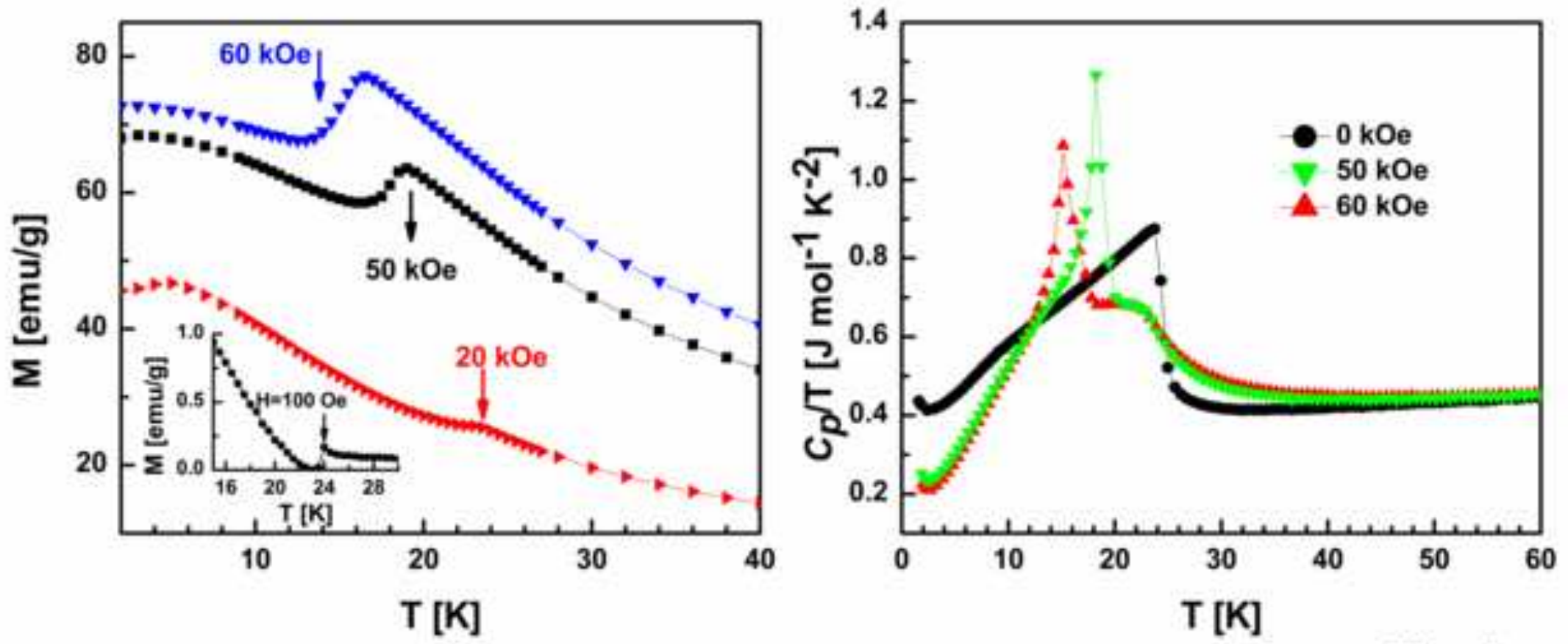

Fig. 1 


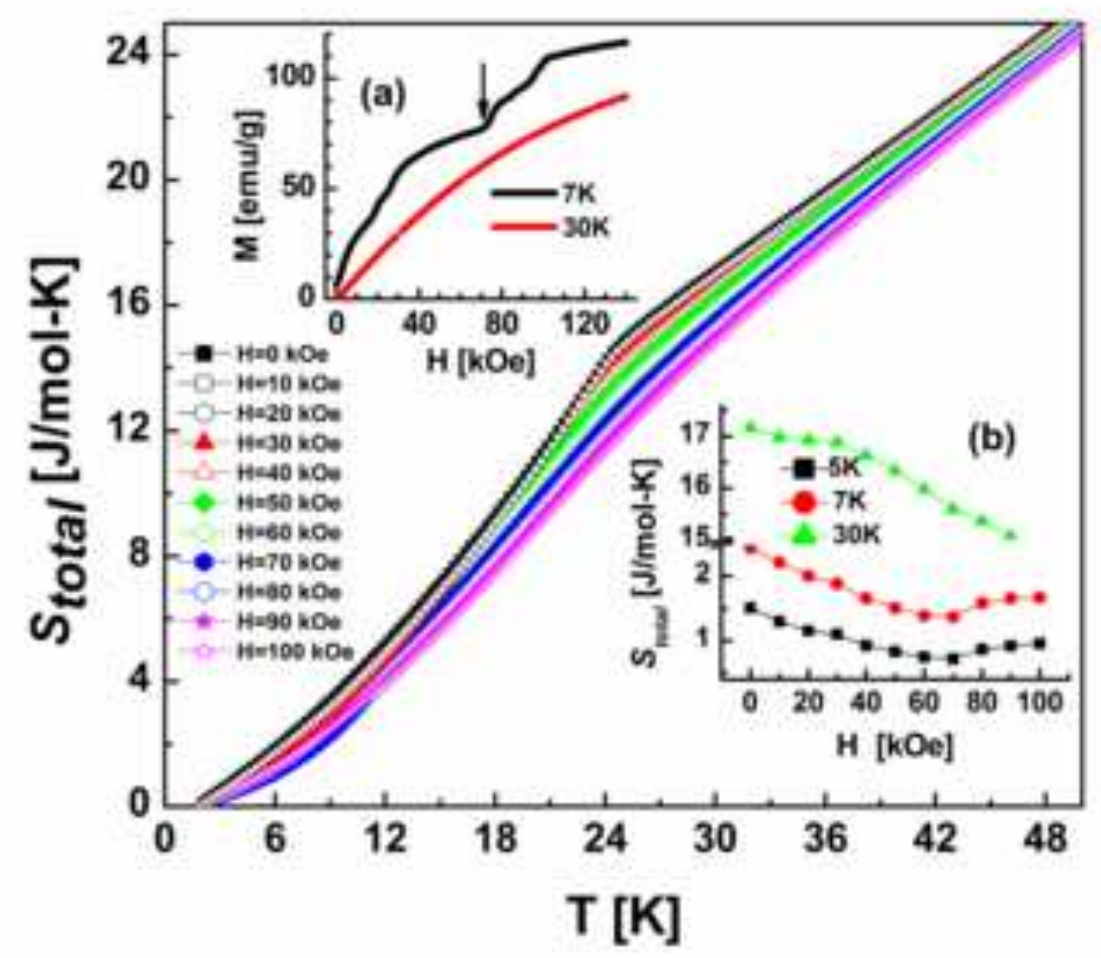

Fig. 2 

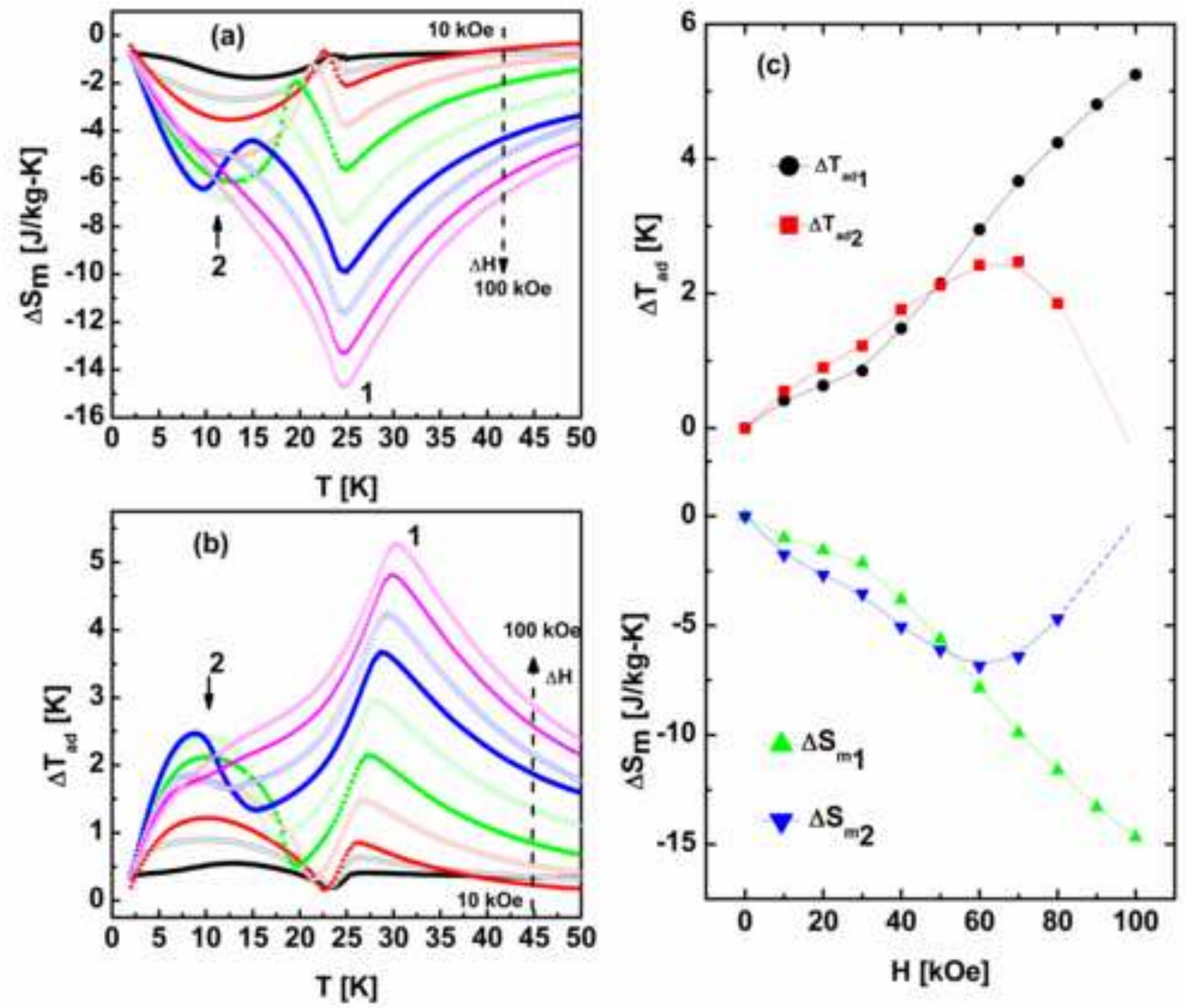

Fig. 3 

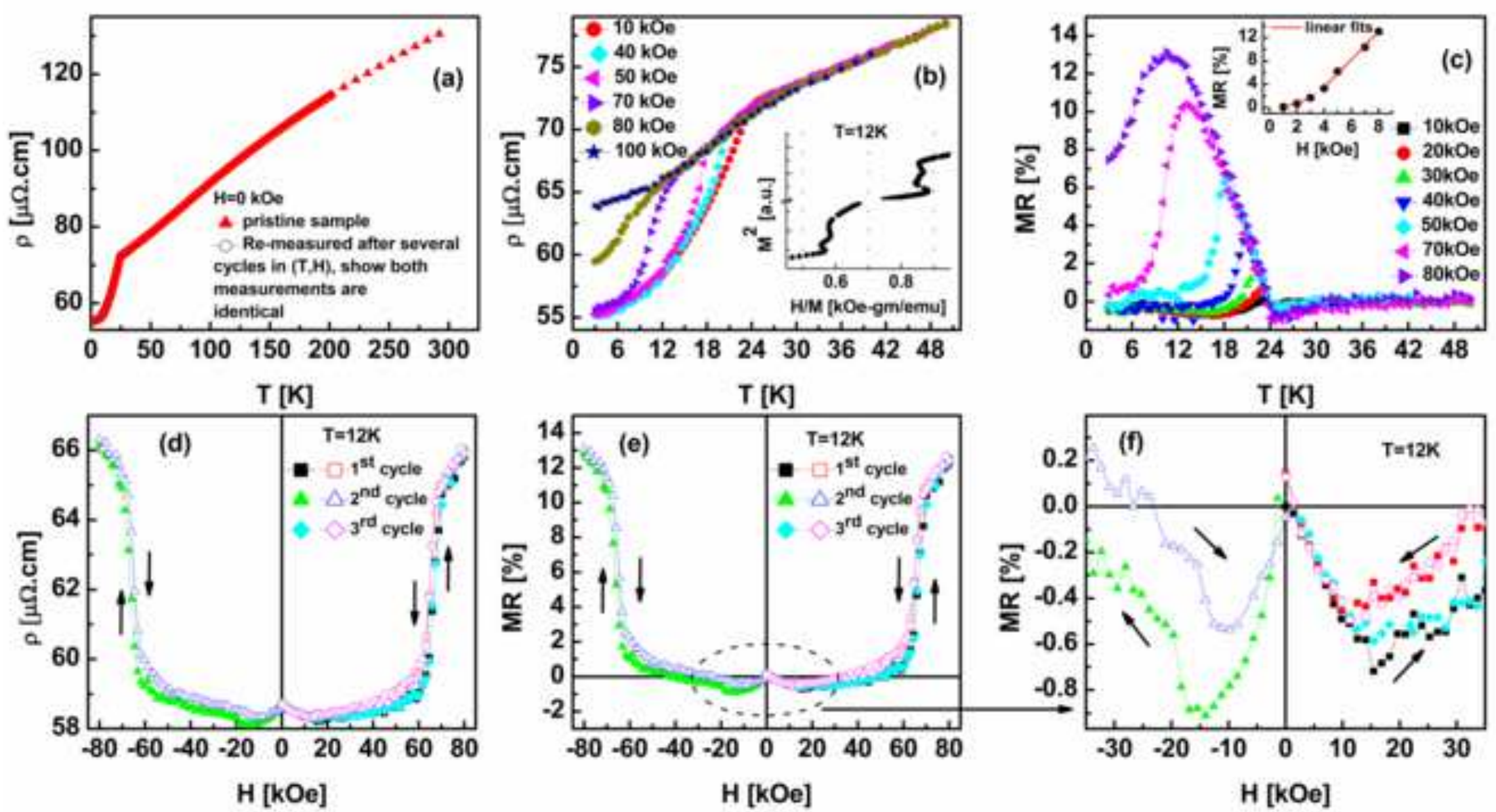

Fig.4 